\title{
Surveillance des expositions en laboratoire aux agents pathogènes humains et aux toxines au Canada en 2016
}

\author{
A Bienek ${ }^{1}, \mathrm{M} \mathrm{Heisz}^{1}, \mathrm{M} \mathrm{Su}^{1 *}$
}

\section{Résumé}

Contexte : Le Canada a récemment adopté un projet de loi autorisant la collecte de données sur les incidents en laboratoire mettant en cause un agent biologique. L'Agence de la santé publique du Canada (l'ASPC) est responsable de cette activité dans le cadre d'un programme national complet visant à protéger les Canadiens des risques pour la santé et la sécurité posés par les agents pathogènes humains et d'animaux terrestres ainsi que les toxines.

Objectif : Présenter les données sur les incidents d'exposition en laboratoire et les infections contractées en laboratoire au Canada, qui ont été recueillies au cours de la première année de collecte de données suivant l'entrée en vigueur du Règlement sur les agents pathogènes humains et les toxines.

Méthodologie : Les incidents survenus entre le 1er janvier et le 31 décembre 2016 ont été déclarés par les parties réglementées du gouvernement fédéral de l'ensemble du Canada au moyen d'un formulaire normalisé du système de surveillance de déclaration des incidents en laboratoire au Canada (DILC). Les incidents d'exposition ont été décrits par secteur, fréquence de survenue, délai de déclaration, nombre de personnes touchées, agents pathogènes humains et toxines en cause, causes et mesures correctives prises. Le programme Microsoft Excel 2010 a été utilisé pour les analyses descriptives de base.

Résultats : En 2016, 46 incidents d'exposition ont été signalés par les titulaires de 835 permis en vigueur au Canada, ce qui représente 1352 zones de confinement autorisés à réaliser des activités comportant la manipulation d'agents pathogènes humains ou de toxines, pour un taux d'incidence globale de $3,4 \%$. Le nombre d'incidents déclarés était le plus élevé dans les secteurs universitaire ( $n=16 ; 34,8 \%$ ) et hospitalier $(n=12 ; 26,1 \%)$, alors qu'il était relativement faible dans le secteur privé. En moyenne, quatre ou cinq incidents ont eu lieu chaque mois; le mois de septembre au cours duquel 10 incidents sont survenus est présenté comme étant une valeur aberrante. Au total, 100 personnes ont été exposées, aucun cas d'exposition indirecte n'a toutefois été signalé. Quatre incidents ont causé des infections soupçonnées $(n=3)$ ou confirmées $(n=1)$ contractées en laboratoire. La plupart des incidents mettaient en cause des agents pathogènes classés dans le groupe de risque 2 ayant été manipulés dans un laboratoire de niveau de confinement $2(91,3 \%)$. Plus de 22 différentes espèces d'agents pathogènes humains et de toxines ont été mises en cause, les bactéries ayant été les plus souvent signalées (34,8\%), suivies des virus (26,1\%). Onze incidents $(23,9 \%)$ se rapportaient à un agent biologique à cote de sécurité élevée (ABCSE). Le non-respect des procédures $(n=15)$ et les blessures par un objet tranchant ou piquant $(n=14)$ étaient les précurseurs les plus courants d'une exposition. Dans 10 cas $(21,7 \%)$, la possession involontaire (c.-à-d. I'isolement d'un agent biologique inattendu lors d'activités habituelles) a joué un rôle. Des améliorations possibles aux procédures d'opération normalisées ont été mentionnées dans $71,7 \%$ des incidents. Des améliorations ont également été notées en ce qui concerne les communications $(26,1 \%)$ et la gestion (23,9\%).

Conclusions : Le système de surveillance de déclaration des incidents en laboratoire au Canada est l'un des premiers systèmes de surveillance au monde, qui recueille des données exhaustives sur les incidents en laboratoire mettant en cause des agents pathogènes humains et des toxines. Les incidents d'exposition signalés au cours de la première année ont été relativement rares, survenant dans moins de $4 \%$ des zones de confinement des laboratoires.

\begin{abstract}
Affiliation
${ }^{1}$ Centre de la biosûreté, Agence de la santé publique du Canada Ottawa (Ontario)
\end{abstract}

\author{
*Correspondance: ming.su@ \\ canada.ca
}

Citation proposée : Bienek A, Heisz M, Su M. Surveillance des expositions en laboratoire aux agents pathogènes humains et aux toxines au Canada en 2016. Relevé des maladies transmissibles au Canada. 2017;43(11):259-68. https://doi.org/10.14745/ccdr.v43i11a04f

\section{Introduction}

L'étude des agents biologiques dans les laboratoires universitaires, vétérinaires, industriels et gouvernementaux comporte de nombreux avantages, bien qu'elle pose un risque inhérent d'exposition en raison de la nature des activités qui y sont réalisées ainsi que des agents pathogènes et des toxines qui y sont manipulés. À l'échelle internationale, ce risque pour les humains lié à la biosécurité et à la biosûreté a entraîné des blessures, des accidents ayant été signalés dans les publications et par les gouvernements (1-4). Quoique rares, des décès sont également survenus $(5,6)$. 
À l'heure actuelle, il existe un nombre limité d'exigences internationales régissant la déclaration des incidents en laboratoire mettant en cause des agents biologiques, et celles-ci diffèrent. En Grande-Bretagne, dans le contexte d'un système de déclaration de plus grande envergure, le Health and Safety Executive impose la déclaration obligatoire des incidents entraînant des maladies causées par des agents biologiques dans un large éventail de milieux de travail (y compris les établissements universitaires, hospitaliers ainsi que du gouvernement central et des gouvernements locaux) (7). En Angleterre, au pays de Galles et en Irlande du Nord, un système de surveillance active a été conçu pour enregistrer les cas d'exposition professionnelle, mais seulement ceux qui sont attribuables à certains virus transmissibles par le sang (8). Autrement, dans la plupart des cas, la déclaration des incidents d'infection contractée en laboratoire se fait de façon volontaire ou dans le cadre d'enquêtes (9-11).

Le Canada dispose de l'un des premiers systèmes de surveillance complets à l'échelle nationale, qui recueille des données des rapports soumis en temps quasi réel sur les incidents relatifs à un large éventail d'agents pathogènes pour les humains et les animaux terrestres et de toxines utilisés en laboratoire. Le système de surveillance de déclaration des incidents en laboratoire au Canada a été officiellement lancé en décembre 2015 en réponse à la sanction de la Loi sur les agents pathogènes humains et les toxines en 2009 et à la promulgation du Règlement sur les agents pathogènes humains et les toxines en 2015, et dans le cadre d'un plus vaste programme national complet de biosûreté et de biosécurité visant à protéger le public canadien des risques pour la santé et la sécurité posés par les agents pathogènes humains et les toxines (12-13). Un aperçu de la portée, des exigences de délivrance de permis pour les laboratoires et du mandat de l'Agence de la santé publique du Canada (I'ASPC) en ce qui concerne la réglementation et la surveillance de l'utilisation des agents pathogènes humains et des toxines peut être trouvé ailleurs que dans le présent document (14). Consultez l'annexe pour obtenir la définition de certains termes couramment utilisés.

Aux termes de la Loi sur les agents pathogènes humains et les toxines adoptée au Canada, les agents pathogènes (y compris les bactéries, les virus, les champignons, les protozoaires et les prions) ainsi que les toxines sont classés en quatre groupes de risque en fonction du niveau de risque qu'ils présentent pour une personne (p. ex. employé de laboratoire) et la collectivité (p. ex. le public canadien) (15). Les facteurs pris en compte comprennent la pathogénicité, la gamme d'hôtes et l'aire de répartition naturelle des agents pathogènes humains et des toxines, la voie d'infection et le mode de transmission, la disponibilité du traitement ou des mesures préventives et les conséquences de la libération des agents pathogènes humains et des toxines dans l'environnement (16). Les activités comportant la manipulation d'agents pathogènes du groupe de risque 1 présentent le risque le plus faible et ne sont pas réglementées au Canada. Parmi les activités visées par la réglementation fédérale, la plupart sont menées sur des agents pathogènes du groupe de risque 2 (92,8 \%). Ces agents pathogènes posent un risque modéré pour les personnes, mais un faible risque pour la santé publique, car ils peuvent causer de graves maladies chez les humains, mais sont peu susceptibles de le faire. Les activités comportant la manipulation d'agents pathogènes du groupe de risque 3 représentent actuellement $6,6 \%$ de toutes les activités réglementées. Ces agents pathogènes posent un risque élevé pour les personnes, mais un faible risque pour la santé publique, car ils sont susceptibles de causer de graves maladies, mais $\mathrm{n}^{\prime}$ ont pas tendance à se propager. Seule une petite proportion des activités réalisées au Canada portent sur les agents pathogènes humains et les toxines de la catégorie restante (groupe de risque $4 ; 0,2 \%$ ) et les agents biologiques à cote de sécurité élevée présents en quantités supérieures aux quantités seuils $(0,5 \%)$ qui font partie d'une catégorie particulière, lesquels posent le plus grand risque pour la santé tant pour les personnes que pour la population.

Le Centre de la biosûreté de l'ASPC est chargé de superviser la surveillance continue des incidents en laboratoire mettant en cause des agents pathogènes humains et des toxines. Les données du système de surveillance de déclaration des incidents en laboratoire au Canada sont fournies par les parties réglementées de partout au Canada, qui admettent qu'un incident a eu lieu et qui sont conscientes qu'ils doivent être déclarés en vertu du Règlement sur les agents pathogènes humains et les toxines (12-15). À l'heure actuelle, quatre types d'incidents doivent être déclarés :

- les expositions et les infections contractées en laboratoire;

- la possession involontaire, la production ou la libération d'un agent pathogène humain ou d'une toxine;

- les agents pathogènes humains ou les toxines perdus, volés ou disparus, y compris les agents biologiques à cote de sécurité élevée qui ne sont pas reçus dans les 24 heures suivant la date et l'heure attendues; et

- les changements ayant une incidence sur le confinement.

Lorsqu'un incident survient, le titulaire de permis doit informer I'ASPC en temps opportun afin de s'assurer que la situation est gérée de façon appropriée (12-15). Pour ce qui est des incidents d'exposition ou d'infection contractée en laboratoire, le rapport de déclaration d'incidents initial est soumis "sans délai " pour se conformer aux exigences de déclaration énoncées dans la Loi sur les agents pathogènes humains et les toxines.

Le rapport initial fournit seulement des données essentielles immédiates sur l'incident, y compris les dates importantes, la cause de l'exposition, les personnes touchées et les agents pathogènes humains et les toxines en cause. Un rapport de suivi est ensuite attendu dans les 15 jours suivant le rapport de déclaration initial lorsqu'il s'agit d'incidents mettant en cause des agents biologiques à cote de sécurité élevée ou dans les 30 jours suivant le rapport de déclaration initial lorsqu'il s'agit de tout autre type d'incident d'exposition ou d'infection contractée en laboratoire. Le rapport de suivi a pour but de fournir des renseignements sur les résultats de l'enquête, y compris le traitement et le suivi de la personne touchée, les causes fondamentales et les mesures correctives visant à réduire le risque d'incidents futurs. Le titulaire de permis ou l'agent de sécurité biologique local dirige l'intervention suivant l'incident avec le soutien de l'ASPC au besoin, jusqu'à ce que le problème soit résolu de façon satisfaisante et que le dossier soit clos.

La déclaration normalisée et systématique permet de documenter les expositions afin de faire des comparaisons entre les incidents et au fil du temps. Une analyse active et collective des incidents déclarés permet de déterminer des profils ou des tendances mettant en évidence des problèmes courants 
ou nouveaux à l'échelle nationale. Fondée sur les données recueillies et stockées dans le système de surveillance de déclaration des incidents en laboratoire au Canada, cette étude fournit un résumé descriptif et une interprétation des données sur les expositions et les infections contractées en laboratoire au Canada, qui ont été recueillies au cours de la première année complète, soit entre le $1^{\text {er }}$ janvier et le 31 décembre 2016.

\section{Méthodologie}

Le système de surveillance de déclaration des incidents en laboratoire au Canada est un " guichet unique " pour la déclaration des incidents par voie électronique. Le système est hébergé dans un système de gestion des relations avec la clientèle de Microsoft Dynamics tenu à jour et sécurisé par une équipe de soutien de la technologie de l'information de I'ASPC. La plupart des champs de données sont obligatoires, et une spécificité élevée est obtenue grâce à l'utilisation d'un formulaire normalisé. Les données saisies dans ce système de surveillance sont autodéclarées, et leur exactitude est validée par le processus d'enquête continu auquel participent l'ASPC et le déclarant. $\mathrm{Si}$, au cours du processus d'enquête, il est considéré qu'un incident ne relève pas de la portée des exigences définies dans la Loi sur les agents pathogènes humains et les toxines, celui-ci est rejeté et exclu de l'analyse.

Les données sur les incidents d'exposition en laboratoire ou d'infection contractée en laboratoire (classés dans les catégories " exposition ", " infection contractée en laboratoire : soupçonnée " ou « infection contractée en laboratoire : confirmée ») survenus en 2016 ont été extraites du système. Les éléments de données comprennent des renseignements sur le permis (nombre de permis, nombre de zones de confinement), le secteur (universitaire, hospitalier, privé/entreprises, santé publique, vétérinaire/santé animale et environnement), les dates importantes (date de l'incident, date de déclaration initiale, date du rapport de suivi), les personnes touchées (nombre de cas d'infection primaire, nombre de cas secondaires), les agents pathogènes humains et les toxines en cause (type, groupe de risque), la cause de l'incident (procédure, objets tranchants et piquants, équipement de protection individuelle, animaux, déversement, insectes, équipement, bris de confinement) et les domaines d'amélioration (procédures d'opération normalisées, formation, communications, gestion et surveillance, équipement, interactions humaines). Microsoft Excel 2010 a été utilisé aux fins des analyses descriptives de base des variables catégoriques (nombres, proportions) et des variables continues (moyenne, intervalle). Puisque les nombreux renseignements recueillis permettent l'identification de l'établissement détenant un permis, les caractéristiques d'identification ont été supprimées, si nécessaire. Toutes les données ont été rapportées, sauf dans les cas où il était possible de reconnaître un incident ou un laboratoire précis.

\section{Résultats}

Pour l'ensemble du Canada, au cours de l'année civile 2016, 835 permis en vigueur permettaient l'utilisation d'agents pathogènes humains et de toxines, ce qui représentait 1352 zones de confinement. Une zone de confinement est une zone physique répondant aux exigences relatives à un certain niveau de confinement requis pour mener des activités liées à la manipulation d'agents pathogènes humains et de toxines particuliers. Un laboratoire peut contenir plusieurs zones de confinement (voir l'annexe pour obtenir une définition complète).

Au total, 50 incidents d'exposition potentielle ont été extraits de la base de données, y compris quatre incidents signalés en 2017, mais survenus en 2016. Au cours du processus d'enquête, il a été établi qu'aucune exposition n'était survenue au cours des quatre incidents; ceux-ci ont été écartés et supprimés de I'analyse, ce qui a porté le total à 46 incidents. L'échantillon comprenait neuf incidents dont la déclaration avait été retardée jusqu'à la délivrance du permis; ceux-ci ont été retenus aux fins de l'analyse, mais ont été exclus des calculs relatifs au délai de déclaration.

Les incidents d'exposition ou d'infection contractée en laboratoire sont survenus à une fréquence de 3,4 \% dans l'ensemble des zones de confinement réglementées. La plupart des incidents déclarés consistaient uniquement en une exposition ( $n=42 ; 91,3 \%$ ), tandis que quatre incidents étaient à l'origine d'une infection contractée en laboratoire soupçonnée ( $n=3 ; 6,5 \%$ ) ou confirmée ( $n=1 ; 2,2 \%$ ). La plupart des incidents mettaient en cause des agents pathogènes humains et des toxines du groupe de risque 2 ayant été manipulés dans un laboratoire de de niveau de confinement 2 (91,3\%). Trois incidents ont eu lieu dans une installation de niveau de confinement 3 et un cas est survenu dans une installation de niveau de confinement 4 .

\section{Répartition des incidents par secteur}

Le plus grand nombre d'incidents déclarés est survenu dans les secteurs universitaire $(n=16 ; 34,8 \%)$ et hospitalier $(n=12$; $26,1 \%$ ), ce qui était proportionnel à la répartition des zones de confinement par secteur (tableau 1). Le secteur privé représentait $32,2 \%$ de toutes les zones de confinement, mais seulement $17,4 \%$ des incidents d'exposition signalés.

Tableau 1 : Incidents d'exposition à un agent pathogène humain ou à une toxine signalés par secteur au Canada en 2016

\begin{tabular}{|c|c|c|c|c|c|c|}
\hline \multirow[t]{2}{*}{ Secteur } & \multicolumn{2}{|c|}{$\begin{array}{l}\text { Nombre } \\
\text { de permis } \\
\text { en vigueur }\end{array}$} & \multicolumn{2}{|c|}{$\begin{array}{c}\text { Nombre } \\
\text { de zones } \\
\text { de confinement }\end{array}$} & \multicolumn{2}{|c|}{$\begin{array}{c}\text { Nombre } \\
\text { d'incidents } \\
\text { d'exposition }\end{array}$} \\
\hline & $\mathbf{n}$ & $\%$ & $\mathbf{n}$ & $\%$ & $\mathbf{n}$ & $\%$ \\
\hline Universitaire & 168 & 20,1 & 436 & 32,2 & 16 & 34,8 \\
\hline Hospitalier & 186 & 22,3 & 290 & 21,4 & 12 & 26,1 \\
\hline Privé/entreprises & 376 & 45,0 & 436 & 32,2 & 8 & 17,4 \\
\hline $\begin{array}{l}\text { Santé publique } \\
\text { (gouvernemental) }\end{array}$ & 25 & 3,0 & 64 & 4,7 & 4 & 8,7 \\
\hline $\begin{array}{l}\text { Vétérinaire/ } \\
\text { santé animale } \\
\text { (gouvernemental) }\end{array}$ & 18 & 2,2 & 38 & 2,8 & 4 & 8,7 \\
\hline $\begin{array}{l}\text { Environnement } \\
\text { (gouvernemental) }\end{array}$ & 32 & 3,8 & 37 & 2,7 & 0 & 0 \\
\hline $\begin{array}{l}\text { Autre secteur } \\
\text { gouvernemental }\end{array}$ & 30 & 3,6 & 51 & 3,8 & 2 & 4,3 \\
\hline TOTAL & 835 & 100 & 1352 & 100 & 46 & 100 \\
\hline
\end{tabular}


LES NOTES DE BASE POUR TABLEAU 1

Abréviation : $n$, nombre

REMAROUES : Les données proviennent du système de surveillance de déclaration des incidents en laboratoire au Canada (extraites le 26 mai 2017)

"Zone de confinement " s'entend d'une zone physique qui répond aux exigences minimales liées au confinement physique et aux pratiques opérationnelles visant la manipulation sécuritair de matières infectieuses et de toxines d'un groupe de risque précis dans les laboratoires et les environnements de travail avec des animaux

Le secteur « universitaire » comprend notamment les universités, les collèges de médecine vétérinaire, les collèges et les cégeps

e secteur u hospitalier ncomprend les hôpitaux affiliés à une université et les hôpitaux non Le secteur " hospitalier La catégorie "secteur privé/entreprises " comprend les industries de la santé humaine, de la santé animale et de la biotechnologie, l'industrie pharmaceutique, l'industrie alimentaire ainsi que les fournisseurs d'agents pathogènes ou de toxines

Le secteur de la "santé publique », le secteur "vétérinaire/santé animale ", le secteur de l'« environnement » et les « autres secteurs » comprennent le gouvernement fédéral, les gouvernements provinciaux et territoriaux et les administrations municipales

\section{Fréquence des incidents et délai de déclaration}

En général, quatre ou cinq incidents ont eu lieu chaque mois, bien que leur nombre soit moins élevé au cours de l'été

(figure 1). Le mois de septembre est présenté en tant que valeur aberrante, 10 incidents d'exposition ayant été signalés à l'ASPC. Après examen, aucun lien n'a été établi entre tous les incidents survenus en septembre et un quelconque emplacement, titulaire de permis ou agent pathogène humain ou toxine en cause. En outre, les caractéristiques des incidents survenus en septembre étaient semblables à celles de tous les autres incidents selon les analyses par niveau de confinement, secteur et type d'agent pathogène.

Figure 1 : Incidents d'exposition à un agent pathogène humain ou à une toxine signalés par mois de survenue de l'incident au Canada en 2016

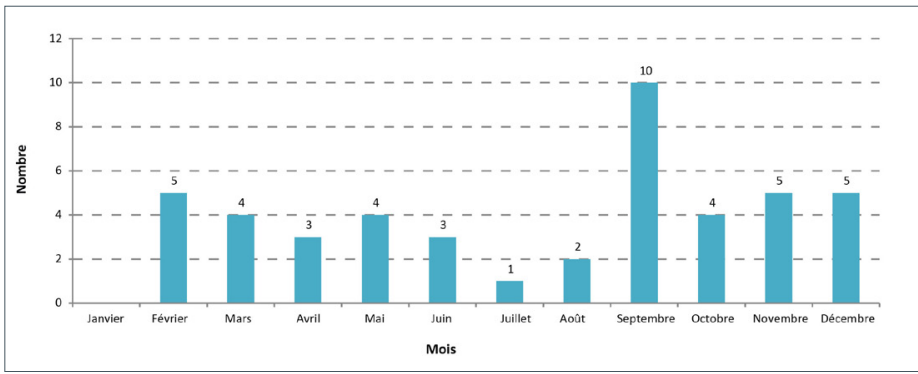

REMARQUE : Les données proviennent du système de surveillance de déclaration des incidents en laboratoire au Canada (extraites le 26 mai 2017)

En ce qui concerne les incidents ne mettant pas en cause un agent biologique à cote de sécurité élevée, le nombre de jours entre la survenue de l'incident et la déclaration initiale de I'incident à I'ASPC variait de 1 à 119 jours, le délai moyen étant de 23,5 jours (selon les jours civils et incluant les jours fériés) (tableau 2). Bien que cela ne figure pas au tableau 2, la moitié des incidents ont été signalés initialement à l'ASPC dans un délai d'environ une semaine suivant leur survenue, alors que neuf incidents ont été déclarés plus d'un mois après leur survenue. Les raisons justifiant un tel retard comprenaient le manque de sensibilisation quant aux exigences en matière de déclaration $(n=4)$ et le besoin d'aide pour soumettre le rapport $(n=3)$. En moyenne, les rapports de suivi ont été soumis 18,4 jours après le rapport initial, $89,3 \%$ des déclarations ayant été soumises dans le délai prévu de 30 jours.
Tableau 2 : Délai de déclaration des incidents d'exposition au Canada en 2016

\begin{tabular}{|c|c|c|c|c|c|c|c|c|c|}
\hline \multirow{2}{*}{$\begin{array}{c}\text { Type } \\
d^{\prime} \text { incident }\end{array}$} & \multicolumn{2}{|c|}{ Intervalle de temps } & \multirow{2}{*}{$\begin{array}{c}\text { Intervalle } \\
\text { cible }\end{array}$} & \multicolumn{2}{|c|}{ Intervalle réel } & \multicolumn{2}{|c|}{$\begin{array}{c}\text { Nombre } \\
\text { d'incidents } \\
\text { déclarés } \\
\text { avant la } \\
\text { date limite }\end{array}$} & \multicolumn{2}{|c|}{$\begin{array}{c}\text { Nombre } \\
\text { d'incidents } \\
\text { déclarés } \\
\text { après la } \\
\text { date limite }\end{array}$} \\
\hline & De & $\grave{A}$ & & $\begin{array}{c}\text { Intervalle } \\
\text { de jours }\end{array}$ & $\begin{array}{c}\text { Nombre } \\
\text { moyen } \\
\text { de jours }\end{array}$ & $\mathrm{n}$ & $\%$ & $\mathrm{n}$ & $\%$ \\
\hline \multirow{2}{*}{$\begin{array}{l}\text { Ne mettant } \\
\text { pas en } \\
\text { cause un } \\
\text { agent } \\
\text { biologique } \\
\text { à cote de } \\
\text { sécurité } \\
\text { élevée* }\end{array}$} & $\begin{array}{l}\text { Survenue } \\
\text { de } \\
\text { l'incident }\end{array}$ & $\begin{array}{l}\text { Déclaration } \\
\text { initiale }\end{array}$ & $\begin{array}{l}\text { Aucun } \\
\text { délai }\end{array}$ & 1 à 119 & 23,5 & S.O. & S.O. & S.O. & S.O. \\
\hline & $\begin{array}{l}\text { Déclaration } \\
\text { initiale† }\end{array}$ & $\begin{array}{l}\text { Rapport de } \\
\text { suivi }\end{array}$ & 30 & 0 à 39 & 18,4 & 25 & 89,3 & 3 & 10,7 \\
\hline \multirow{2}{*}{$\begin{array}{l}\text { Mettant } \\
\text { en cause } \\
\text { un agent } \\
\text { biologique } \\
\text { à cote de } \\
\text { sécurité } \\
\text { élevée }\end{array}$} & $\begin{array}{l}\text { Survenue } \\
\text { de } \\
\text { l'incident }\end{array}$ & $\begin{array}{l}\text { Déclaration } \\
\text { initiale }\end{array}$ & $\begin{array}{l}\text { Aucun } \\
\text { délai }\end{array}$ & 0 à 65 & 17,1 & S.O. & S.O. & S.O. & S.O. \\
\hline & $\begin{array}{l}\text { Déclaration } \\
\text { initiale† }\end{array}$ & $\begin{array}{l}\text { Rapport de } \\
\text { suivi }\end{array}$ & 15 & 0 à 39 & 15,9 & 7 & 77,4 & 2 & 22,2 \\
\hline
\end{tabular}

Abréviations : $n$, nombre; S.O., sans objet

REMARQUES : Les données proviennent du système de surveillance de déclaration des incidents en laboratoire au Canada (extraites le 26 mai 2017)

Selon les jours civils (y compris les jours fériés) entre la notification initiale et le premier rapport de suivi

Exclut $\mathrm{n}=9$ incidents qui ne pouvaient pas être signalés avant la délivrance du permis

Comprend les incidents pour lesquels l'agent biologique est inconnu $(n=5)$

Déclaration initiale à l'ASPC tel qu'il est requis par les lois de réglementation

En ce qui concerne les incidents mettant en cause un agent biologique à cote de sécurité élevée, le nombre de jours entre la survenue de l'incident et la déclaration initiale de l'incident à I'ASPC variait de 0 à 65 jours, le délai moyen étant de 17,1 jours (selon les jours civils et incluant les jours fériés) (tableau 2). Le délai pour soumettre un rapport de suivi après la déclaration initiale était de 15 jours; 77,4 \% des rapports de suivi ont été soumis dans ce délai. Aucune raison claire n'a été fournie pour expliquer le retard de déclaration dans le cas de deux incidents soumis après le délai prévu. II est courant de noter de tels délais de déclaration avec les nouveaux systèmes de réglementation et ces délais sont réduits à mesure que les parties réglementées sont informées de leurs obligations en matière de déclaration.

\section{Nombre de personnes touchées}

À la suite des 46 incidents, 100 personnes ont été exposées à un agent pathogène humain ou à une toxine. Dans la plupart des cas $(84,8 \%)$, une seule personne a été exposée, tandis qu'au cours de deux incidents, deux personnes ont été exposées et qu'au cours de cinq incidents, trois personnes ou plus ont été exposées. Tous les incidents au cours desquels deux personnes ou plus ont été exposées sont survenus en milieu hospitalier ou dans des laboratoires de diagnostic. Des 100 personnes touchées, quatre ont reçu un diagnostic d'infection contractée en laboratoire soupçonnée ou confirmée. Aucun cas d'exposition indirecte n'a été signalé. 


\section{Agents pathogènes humains et toxines en cause}

Parmi plus de 22 différentes espèces d'agents pathogènes humains et de toxines en cause dans les incidents, les bactéries étaient celles qui étaient les plus fréquemment signalées, plus de 16 incidents (34,8\%) étant liés à une bactérie du groupe de risque $2(n=14)$ ou du groupe de risque $3(n=2)$, à l'exception des bactéries classées en tant qu'agents biologiques à cote de sécurité élevée (tableau 3). Au total, 11 incidents (23,9\%) mettaient en cause un agent biologique à cote de sécurité élevée du groupe de risque $3(n=10)$ ou du groupe de risque 4 $(n=1)$. L'agent pathogène humain ou la toxine le plus souvent signalé $(n=5)$ était l'espèce bactérienne Brucella spp., qui est classée en tant qu'agent biologique à cote de sécurité élevée du groupe de risque 3 .

Tableau 3 : Agents pathogènes humains ou toxines en cause signalés dans les incidents d'exposition par groupe de risque et type d'agent biologique au Canada en 2016

\begin{tabular}{|c|c|c|c|c|c|c|c|c|c|c|}
\hline \multirow{2}{*}{$\begin{array}{c}\text { Type } \\
\text { d'agent } \\
\text { biologique }\end{array}$} & \multicolumn{2}{|c|}{$\begin{array}{c}\text { Groupe } \\
\text { de } \\
\text { risque } 2\end{array}$} & \multicolumn{2}{|c|}{$\begin{array}{c}\text { Groupe } \\
\text { de } \\
\text { risque } 3\end{array}$} & \multicolumn{2}{|c|}{$\begin{array}{l}\text { Groupe } \\
\text { de } \\
\text { risque } 4\end{array}$} & \multicolumn{2}{|c|}{ Inconnu } & \multicolumn{2}{|c|}{ Total } \\
\hline & $n$ & $\%$ & $n$ & $\%$ & $n$ & $\%$ & $\mathbf{n}$ & $\%$ & $\mathrm{n}$ & $\%$ \\
\hline Bactérie & 14 & 51,9 & 2 & 15,4 & 0 & 0 & 0 & 0 & 16 & 34,8 \\
\hline Virus & 11 & 40,7 & 1 & 7,7 & 0 & 0 & 0 & 0 & 12 & 26,1 \\
\hline Champignon & 1 & 3,7 & 0 & 0 & 0 & 0 & 0 & 0 & 1 & 2,2 \\
\hline Parasite & 1 & 3,7 & 0 & 0 & 0 & 0 & 0 & 0 & 1 & 2,2 \\
\hline $\begin{array}{l}\text { Agent } \\
\text { biologique } \\
\text { à cote de } \\
\text { sécurité } \\
\text { élevée }\end{array}$ & 0 & 0 & 10 & 76,9 & 1 & 100 & 0 & 0 & 11 & 23,9 \\
\hline Inconnu & 0 & 0 & 0 & 0 & 0 & 0 & 5 & 100 & 5 & 10,9 \\
\hline TOTAL & 27 & 100 & 13 & 100 & 1 & 100 & 5 & 100 & 46 & 100 \\
\hline
\end{tabular}

REMARQUES : nem déclaration des incidents en laboratoire au Canada (extraites le 30 mai 2017)

Les activités comportant la manipulation d'agents pathogènes humains et de toxines du groupe de risque 1 ne sont pas réglementées par le gouvernement fédéral

Les " agents biologiques à cote de sécurité élevée » en cause dans les incidents déclarés comprennent des bactéries et des virus

La catégorie « inconnu » comprend les incidents pour lesquels l'agent biologique en cause n'a jamais été identifié

\section{Causes des incidents}

Bien que les causes les plus fréquentes à l'origine d'un incident aient été le non-respect des procédures $(n=15)$ et les objets tranchants et piquants ( $n=14$ ) (figure 2 ), des problèmes liés à l'équipement de protection individuelle, à la manipulation des animaux, aux déversements, à l'équipement et au bris de confinement ont également été mentionnés. Après l'examen des incidents, il a été établi que sept cas signalés dans la catégorie " autre » auraient été probablement mieux classés dans une ou plusieurs catégories existantes. Plus particulièrement, dans 10 cas $(21,7 \%)$, la possession involontaire ou l'isolement d'un agent biologique pendant des activités menées régulièrement a joué un rôle dans l'exposition (données non présentées). Puisque les titulaires de permis pour le groupe de risque 2 sont seulement autorisés à mener des activités comportant la manipulation d'agents pathogènes humains et de toxines du groupe de risque 2 présents en quantités inférieures aux quantités seuils, un incident au cours duquel tout agent pathogène humain ou toxine du groupe de risque 3 ou 4 présent en quantité supérieure à la quantité seuil avec lequel un titulaire de permis entrerait en contact serait considéré comme étant une possession involontaire et pourrait poser un risque accru pour le personnel.

Figure 2 : Causes signalées des incidents d'exposition à un agent pathogène humain ou à une toxine au Canada en 2016

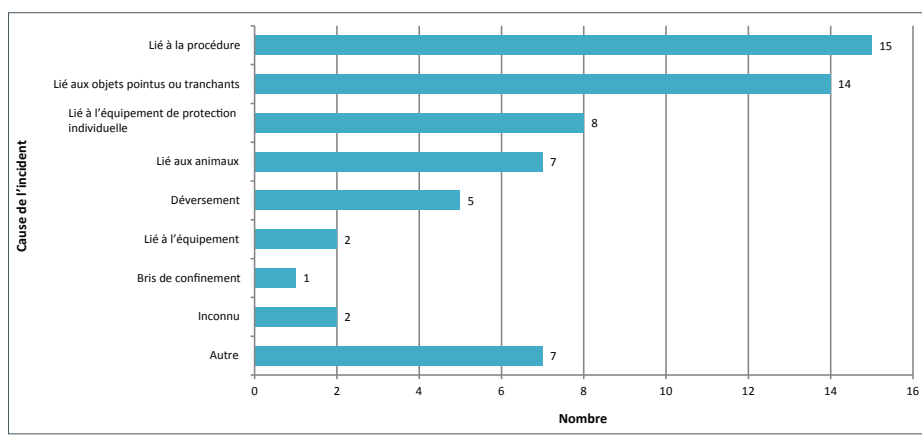

REMARQUES : Les données proviennent du système de surveillance de déclaration des incidents en laboratoire au Canada (extraites le 26 mai 2017)

La catégorie " objets tranchants et piquants » comprend les piqûres d'aiguille et autres blessures par objets tranchants et piquants

La catégorie « équipement de protection individuelle » comprend un équipement de protection individuelle inapproprié ou la défaillance d'un tel équipement

La catégorie " animaux » comprend les morsures et les égratignures

\section{Mesures correctives visant à améliorer la sécurité dans les laboratoires}

À la suite du processus d'enquête, la plupart des déclarants ont été en mesure de déterminer les causes fondamentales ou les éléments des systèmes et des processus à améliorer qui permettraient d'éviter un incident semblable à l'avenir. Les éléments à améliorer les plus souvent mentionnés se rapportaient aux normes, aux procédures d'opération normalisées, aux politiques, aux règles et aux procédures électroniques (71,7 \%) (tableau 4). Des problèmes relatifs aux communications ont été mis en cause dans le quart des incidents $(26,1 \%)$ et des problèmes liés à la gestion ou à la supervision ont été cités dans 11 incidents (23,9\%). Au moment de l'analyse, les 15 causes fondamentales de la catégorie " autre " auraient pu être classées dans les catégories existantes, car les problèmes mentionnés étaient liés aux communications et à l'équipement ou attribuables à l'erreur humaine. 
Tableau 4 : Éléments à améliorer relativement aux incidents d'exposition à un agent pathogène humain ou à une toxine signalés au Canada en 2016

\begin{tabular}{|c|c|c|c|}
\hline \multirow{2}{*}{$\begin{array}{c}\text { Cause } \\
\text { fondamentale }\end{array}$} & \multirow[t]{2}{*}{ Préoccupations } & $\begin{array}{l}\text { Men- } \\
\text { tions }\end{array}$ & Proportion' \\
\hline & & $\mathbf{n}$ & $\%$ \\
\hline \multirow[t]{5}{*}{$\begin{array}{l}\text { Procédures } \\
\text { d'opération } \\
\text { normalisées }\end{array}$} & $\begin{array}{l}\text { Les procédures étaient } \\
\text { connues, mais n'ont pas } \\
\text { été suivies }\end{array}$ & \multirow{5}{*}{33} & \multirow{5}{*}{71,7} \\
\hline & $\begin{array}{l}\text { Les procédures } \\
\text { n'étaient pas connues } \\
\text { de l'utilisateur }\end{array}$ & & \\
\hline & $\begin{array}{l}\text { Les procédures n'ont } \\
\text { pas été correctement } \\
\text { suivies }\end{array}$ & & \\
\hline & $\begin{array}{l}\text { Les procédures ne } \\
\text { correspondaient pas à } \\
\text { la tâche ou à l'activité }\end{array}$ & & \\
\hline & $\begin{array}{l}\text { Les procédures } \\
\text { n'étaient pas } \\
\text { développées et mises } \\
\text { en œuvre, mais auraient } \\
\text { dû l'être }\end{array}$ & & \\
\hline \multirow[t]{4}{*}{ Formation } & $\begin{array}{l}\text { La formation n'avait pas } \\
\text { été élaborée ni mise en } \\
\text { œuvre }\end{array}$ & \multirow{4}{*}{7} & \multirow{4}{*}{15,2} \\
\hline & $\begin{array}{l}\text { La formation était } \\
\text { inappropriée ou n'a pas } \\
\text { suffi }\end{array}$ & & \\
\hline & $\begin{array}{l}\text { La formation a été } \\
\text { offerte, mais n'a pas été } \\
\text { terminée }\end{array}$ & & \\
\hline & $\begin{array}{l}\text { Le personnel n'avait } \\
\text { pas les qualifications } \\
\text { ni les compétences } \\
\text { nécessaires pour mener } \\
\text { à bien la tâche }\end{array}$ & & \\
\hline \multirow[t]{3}{*}{ Communication } & $\begin{array}{l}\text { Aucun système } \\
\text { ou méthode de } \\
\text { communication }\end{array}$ & \multirow{3}{*}{12} & \multirow{3}{*}{26,1} \\
\hline & Aucune communication & & \\
\hline & $\begin{array}{l}\text { La communication était } \\
\text { imprécise, ambiguë ou } \\
\text { incomprise }\end{array}$ & & \\
\hline \multirow[t]{5}{*}{$\begin{array}{l}\text { Gestion et } \\
\text { surveillance }\end{array}$} & $\begin{array}{l}\text { Amélioration nécessaire } \\
\text { de la supervision }\end{array}$ & \multirow{5}{*}{11} & \multirow{5}{*}{23.9} \\
\hline & $\begin{array}{l}\text { Amélioration nécessaire } \\
\text { en ce qui concerne la } \\
\text { vérification, l'évaluation } \\
\text { ou l'application des } \\
\text { procédures d'opération } \\
\text { normalisées }\end{array}$ & & \\
\hline & $\begin{array}{l}\text { Amélioration nécessaire } \\
\text { en ce qui concerne la } \\
\text { vérification, l'évaluation } \\
\text { ou la mise en œuvre de } \\
\text { la formation }\end{array}$ & & \\
\hline & $\begin{array}{l}\text { Amélioration nécessaire } \\
\text { de la préparation }\end{array}$ & & \\
\hline & $\begin{array}{l}\text { Amélioration nécessaire } \\
\text { en ce qui concerne les } \\
\text { facteurs humains }\end{array}$ & & \\
\hline
\end{tabular}

Tableau 4 : Éléments à améliorer relativement aux incidents d'exposition à un agent pathogène humain ou à une toxine signalés au Canada en 2016 (suite)

\begin{tabular}{|c|c|c|c|}
\hline \multirow{2}{*}{$\begin{array}{c}\text { Cause } \\
\text { fondamentale }\end{array}$} & \multirow[t]{2}{*}{ Préoccupations } & $\begin{array}{l}\text { Men- } \\
\text { tions }\end{array}$ & Proportion' \\
\hline & & $n$ & $\%$ \\
\hline \multirow[t]{2}{*}{$\begin{array}{l}\text { Gestion et } \\
\text { surveillance } \\
\text { (suite) }\end{array}$} & $\begin{array}{l}\text { Amélioration nécessaire } \\
\text { en ce qui concerne } \\
\text { l'évaluation des risques }\end{array}$ & \multirow{2}{*}{11} & \multirow{2}{*}{23.9} \\
\hline & $\begin{array}{l}\text { Amélioration nécessaire } \\
\text { en ce qui concerne la } \\
\text { sélection des travailleurs }\end{array}$ & & \\
\hline \multirow[t]{5}{*}{ Équipement } & $\begin{array}{l}\text { Amélioration nécessaire } \\
\text { en ce qui concerne } \\
\text { la conception de } \\
\text { l'équipement }\end{array}$ & \multirow{5}{*}{8} & \multirow{5}{*}{17,4} \\
\hline & $\begin{array}{l}\text { L'équipement n'a } \\
\text { pas été entretenu } \\
\text { correctement }\end{array}$ & & \\
\hline & $\begin{array}{l}\text { Défaillance de } \\
\text { l'équipement }\end{array}$ & & \\
\hline & $\begin{array}{l}\text { L'équipement utilisé } \\
\text { n'était pas approprié } \\
\text { pour la tâche effectuée }\end{array}$ & & \\
\hline & $\begin{array}{l}\text { Le contrôle de la qualité } \\
\text { n'a pas été effectué ou } \\
\text { doit être amélioré }\end{array}$ & & \\
\hline \multirow[t]{3}{*}{$\begin{array}{l}\text { Interactions } \\
\text { humaines }\end{array}$} & $\begin{array}{l}\text { Amélioration } \\
\text { nécessaire en ce qui } \\
\text { concerne l'étiquetage, } \\
\text { la disposition, le } \\
\text { fonctionnement et } \\
\text { l'affichage des outils et } \\
\text { de l'équipement }\end{array}$ & \multirow{3}{*}{8} & \multirow{3}{*}{17,4} \\
\hline & $\begin{array}{l}\text { Amélioration } \\
\text { nécessaire en ce qui } \\
\text { concerne les facteurs } \\
\text { environnementaux à } \\
\text { l'intérieur du milieu de } \\
\text { travail }\end{array}$ & & \\
\hline & $\begin{array}{l}\text { Amélioration nécessaire } \\
\text { en ce qui concerne } \\
\text { les contraintes liées à } \\
\text { la charge de travail, } \\
\text { au stress causé par la } \\
\text { pression ou à d'autres } \\
\text { exigences }\end{array}$ & & \\
\hline \multicolumn{2}{|l|}{ Autre } & 15 & 32,6 \\
\hline
\end{tabular}

Abréviation : n, nombre

'Proportion d'incidents pour lesquels une cause fondamentale a été indiquée

REMARQUES : Plus d'une cause fondamentale peut être indiquée pour un incident. Les données proviennent du système de surveillance de déclaration des incidents en laboratoire au Canada (extraites le 26 mai 2017)

"Procédures d'opération normalisées " s'entend des normes, des politiques, des procédures ou d'autres documents sur la pratique qui orientent les travaux ou les activités

\section{Discussion}

Il s'agit du premier rapport du premier système de surveillance complet à l'échelle nationale sur les expositions en laboratoire aux agents pathogènes humains et aux toxines. Dans l'ensemble, le nombre d'expositions en laboratoire aux agents pathogènes humains et aux toxines était faible. Au cours de la première 
année de collecte de données selon la réglementation exigeant la déclaration obligatoire des incidents, 46 incidents d'exposition ont été signalés. Parmi les cent travailleurs exposés à un agent pathogène humain ou à une toxine, on a dénombré quatre cas d'infection contractée en laboratoire soupçonnée ou confirmée. Aucun cas d'exposition indirecte n'a été déclaré ailleurs que dans les laboratoires. Ces résultats, y compris le nombre maximal d'incidents survenus en septembre et le nombre plus élevé d'incidents survenus dans les laboratoires universitaires, devront être évalués de façon approfondie au cours des prochaines années de collecte de données. Bon nombre des principaux résultats confirment ce qui ressort des publications, c'est-à-dire que les agents biologiques en cause étaient principalement des bactéries $(1,17,18)$, Brucella spp. étant souvent la cause déclarée des infections contractées en laboratoire $(2,19,20)$. En outre, les causes courantes d'exposition étaient une manipulation incorrecte des objets tranchants et piquants ou la possession involontaire d'un agent pathogène humain ou d'une toxine. Ces causes ont également été fréquemment décrites ailleurs que dans le présent document (21-25).

La force de cette recherche vient du fait qu'elle est fondée sur un système de déclaration obligatoire comportant des champs de données normalisés et souvent obligatoires. La recherche comporte toutefois certaines limites qui doivent aussi être prises en considération. Il est peu probable que les données pour 2016 comprennent tous les incidents à déclaration obligatoire en raison de plusieurs facteurs. Tout d'abord, le système en était qu'à ses tout débuts alors que les permis étaient délivrés de façon continue tout au long de l'année de collecte de données. En raison des biais d'auto-sélection ou des biais liés aux non-réponses, il est également possible que les données soient incomplètes, puisque certains incidents n'ont pas été déclarés, ce qui peut comprendre des incidents non détectés et des incidents non déclarés en raison d'un manque de sensibilisation ou manque de compréhension à l'égard des exigences réglementaires ou en raison de la réticence à les déclarer en raison de la connotation négative associée aux " accidents » et aux « incidents ». Les données déclarées peuvent comporter certains biais. Les données autodéclarées peuvent être influencées par de nombreux facteurs, y compris les biais de mémoire, le mode de collecte de données, l'expérience du déclarant ou du personnel et les biais des enquêtés-substituts. Les biais de mémoire seraient particulièrement importants lorsque de nouveaux renseignements sont obtenus ou que des symptômes surviennent, ce qui force les déclarants à travailler à contre-courant pour déterminer l'incident probable ayant mené au résultat. Des changements sont constamment apportés au système de surveillance de déclaration des incidents en laboratoire au Canada par souci de clarté pour les déclarants dans le but d'améliorer le délai de déclaration et la normalisation des données.

Les renseignements tirés de ces données peuvent être utilisés comme point de référence pour éclairer les chercheurs, les parties réglementées et le public sur le contexte actuel de la biosécurité en laboratoire au Canada et le rendement du système de surveillance de déclaration des incidents en laboratoire au Canada à ce jour. Les conclusions sur la qualité des données peuvent servir à orienter l'élaboration de systèmes de surveillance semblables ailleurs dans le monde, tandis que les données elles-même peuvent être utilisées à l'interne par I'ASPC afin de faire respecter les normes de sécurité, d'améliorer les stratégies de prévention et de promouvoir les pratiques exemplaires. En se fondant sur ces résultats généralisés, I'ASPC a déjà mis en œuvre des initiatives de sensibilisation pour accroître la sensibilisation à l'égard des incidents survenant fréquemment, y compris un avis envoyé aux intervenants sur les blessures par objet tranchant ou piquant liées à l'utilisation de lames de scalpel jetables (Bulletin d'information sur la biosécurité et de biosûreté pour les agents pathogènes humains et les toxines - Utilisez-vous des scalpels à lame jetable? mai 2017, bulletin d'information non publié). L'Agence a aussi publié une lettre d'information sur la tendance à la hausse du nombre d'isolements inattendus de Coccidioides spp., peut-être en raison du nombre accru de voyageurs revenant au Canada après un voyage dans le sud-ouest des États-Unis, le nord du Mexique ainsi qu'en Amérique centrale et en Amérique du Sud (Bulletin d'information sur la biosécurité et de biosûreté pour les agents pathogènes humains et les toxines - Rapport du système de déclaration des incidents en laboratoire au Canada - Coccidioides, septembre 2016, bulletin d'information non publié).

\section{Conclusion}

Au Canada, la Loi sur les agents pathogènes humains et les toxines et le Règlement sur les agents pathogènes humains et les toxines exigent la déclaration obligatoire en temps quasi réel des incidents d'exposition en laboratoire aux agents pathogènes humains et aux toxines. Les exigences en matière de déclaration obligatoire appuient la collecte de données exhaustives, opportunes et normalisées. La déclaration des incidents à un organisme fédéral contribue à un objectif plus vaste, soit celui de renforcer la biosécurité et la biosûreté dans les laboratoires canadiens grâce à la compréhension des risques potentiels liés à la pratique, qui pourrait entraîner des changements globaux dont pourraient bénéficier toutes les parties réglementées.

\section{Déclaration des auteurs}

Les auteurs A. Bienek, M. Heisz et M. Su ont participé à la surveillance des incidents en laboratoire. Ils ont travaillé ensemble à la conceptualisation. A. Bienek a préparé l'ébauche initiale, tandis que les trois auteurs ont participé à l'examen des différentes ébauches et ont approuvé la version définitive. M. Heisz et M. Su ont également assumé un rôle de supervision.

\section{Conflit d'intérêt}

Aucun.

\section{Remerciements}

Nous tenons à remercier Ken Turcotte, Ismahan Hussein, Cindy Evans, Craig Brooks, Marnie Fiebig et Jennifer Mihowich du Centre de la biosûreté pour leur expertise et la transmission de données supplémentaires. Nous tenons également à remercier tous les titulaires de permis et les agents de la sécurité biologique de partout au Canada pour la soumission de rapports de haute qualité. 


\section{Financement}

Ce travail a été appuyé par l'Agence de la santé publique du Canada dans le cadre de son mandat de base.

\section{Références}

1. Harding AL, Brandt Byers K. Epidemiology of LaboratoryAssociated Infections. In: Fleming DO, Hunt DL, editors. Biological Safety: Principles and Practices, 4th Edition. Washington DC: ASM Press; 2006. p. 53-77. DOI (http:// dx.doi.org/10.1128/9781555815899).

2. Coelho AC, Diez JG. Biological Risks and LaboratoryAcquired Infections: A Reality that Cannot be Ignored in Health Biotechnology. Front Bioeng Biotechnol 2015. 3(56):1-10. DOI (http://dx.doi.org/10.3389/ fbioe.2015.00056). PubMed (https://www.ncbi.nlm.nih. gov/entrez/query.fcgi?cmd=Retrieve \&db=PubMed\&list_ uids $=25973418 \&$ dopt=Abstract).

3. Centers for Disease Control and Prevention. Human Salmonella Typhimurium Infections Linked to Exposure to Clinical and Teaching Microbiology Laboratories (Final Update). Atlanta: Centers for Disease Control and Prevention; 2014. https://www.cdc.gov/salmonella/ typhimurium-labs-06-14/index.html

4. Centers for Disease Control and Prevention. Human Salmonella Typhimurium Infections Linked to Exposure to Clinical and Teaching Microbiology Laboratories. Atlanta: Centers for Disease Control and Prevention; 2017. https:// www.cdc.gov/salmonella/typhimurium-07-17/index.html

5. Centers for Disease Control and Prevention (CDC). Fatal Laboratory-Acquired Infection with an Attenuated Yersinia Pestis Strain. MMWR Morb. Mortal. Wkly. Rep. 2011;60(7):201-5. PubMed (https://www.ncbi.nlm.nih.gov/pubmed/21346706?do $\mathrm{pt}=$ Abstract).

6. Hawkes N. Smallpox Death in Britain Challenges Presumption of Laboratory Safety. Science. 1979 March 2;203(4383):855- 6. DOI (http://dx.doi.org/10.1126/science.419409). PubMed (https://www.ncbi.nlm.nih.gov/entrez/query.fcgi?cmd=Retrieve $\& d b=$ PubMed\&list_uids=419409\&dopt=Abstract).

7. RIDDOR - Reporting of Injuries, Diseases and Dangerous Occurrences Regulations. London: Health and Safety Executive; 2013. http://www.hse.gov.uk/riddor/

8. Health Protection Agency. Eye of the Needle: United Kingdom Surveillance of Significant Occupational Exposures to Blood Borne Viruses in Healthcare Workers. London: Health Protection Agency; 2006. http://webarchive.nationalarchives. gov.uk/20140714113638/http://www.hpa.org.uk/webc/ HPAwebFile/HPAweb_C/1205394781623

9. Wurtz N, Papa A, Hukic M, Di Caro A, Leparc-Goffart I, Leroy E, Landini MP, Sekeyova Z, Drumler JS, Badescu D, Busquets N, Calistri A, Parolin C, Palu G, Christova I, Maurin M, La Scola B, Raoult D. Survey of Laboratory-Acquired Infections Around the World in Biosafety Level 3 and 4 Laboratories. Eur. J. Clin. Microbiol. Infec. Dis. 2016;35(8):1247-58. DOI (http://dx.doi. org/10.1007/s10096-016-2657-1). PubMed (https://www.ncbi. $\mathrm{nlm}$.nih.gov/entrez/query.fcgi?cmd=Retrieve\&db=PubMed\&li st_uids=27234593\&dopt=Abstract).
10. Willemarck N, Van Vaerenbergh B, Descamps E, Brosius B, Dai Do Thi C, Leunda A, Baldo A. Survey of LaboratoryAcquired Infections in Belgium (2007-2012): An Online Survey. Wetenschappelijk Instituut Volkgezondheid. 2015 February;1-49. http://www.biosafety.be/CU/PDF/2015_Willemarck_LAI\%20 report\%20Belgium_2007_2012_Final.pdf

11. Singh K, Weinstein RA. Laboratory-Acquired Infections. Clinical Infectious Diseases. 2009 July 1;49(1):142-7. DOI (https:// doi.org/10.1086/599104). PubMed (https://www.ncbi.nlm. nih.gov/entrez/query.fcgi?cmd=Retrieve $\& d b=$ PubMed\&lis t_uids=19480580\&dopt=Abstract).

12. Gouvernement du Canada. Réglement sur les agents pathogènes humains et les toxines, DORS/2015/44, Permis (1 er décembre 2015). http://laws.justice.gc.ca/fra/reglements/ DORS-2015-44/page-1.html\#h-2

13. Agence de la santé publique du Canada. Norme canadienne sur la biosécurité: pour les installations où on manipule ou entrepose des agents pathogènes qui touchent les humains et les animaux terrestres, et des toxines. Deuxéieme édition. Ottawa ON: ASPC. 2015. https://www.canada.ca/content/ $\mathrm{dam} / \mathrm{phac}-\mathrm{asp}$ /migration/cbsg-nldcb/cbs-ncb/assets/pdf/ cbsg-nldcb-fra.pdf

14. Labrie C, Lecordier S. Supervision réglementaire des agents pathogènes pour les humains et des toxines au Canada. Relevé des maladies transmissible au Canada. 41(S6):1520. https://www.canada.ca/fr/sante-publique/services/ rapports-publications/releve-maladies-transmissibles-canadarmtc/numero-mensuel/2015-41/rmtc-volume-41s-6-17decembre-2015/rmtc-volume-41s-6-17-decembre-2015-3. html

15. Gouvernement du Canada. Loi sur les agents pathogènes humains et les toxines, L.C. 2009, ch. 24, Obligation d'aviser le ministre (December 1, 2015). http://laws.justice.gc.ca/fra/ lois/H-5.67/page-2.html\#h-10

16. Gouvernement du Canada. Guide canadien sur la biosécurité, Deuxième édition. Ottawa: ASPC. 2016. https://www.canada. $\mathrm{ca} / \mathrm{fr} /$ sante-publique/services/normes-lignes-directricescanadiennes-biosecurite/guide-deuxieme-edition.html

17. Pike RM. Laboratory-Associated Infections: Summary and Analysis of 3921 Cases. Health Lab Sci. 1976;13(2):105-14. PubMed (https://www.ncbi.nlm.nih.gov/entrez/query.fcgi?cmd= Retrieve\&db=PubMed\&list_uids=946794\&dopt=Abstract).

18. Lacroix G. Literature Review of Laboratory-Acquired Infections in Canada and the United-States between 2000 and 2009. Office of Laboratory Security, Public Health Agency of Canada. 2009. http://biosafety.icid.com/en/files/presentations/LiteratureReview-\%20Laboratory-Acquired-Infections-CanadaUS-2000-2009.pdf

19. Traxler RM, Lehman MW, Bosserman EA, Guerra MA, Smith TL. A Literature Review of Laboratory-Acquired Brucellosis. J Clin Microbiol. 2013;51(9): 3055-62. DOI (dx.doi.org/10.1128/ JCM.00135-13). PubMed (https://www.ncbi.nlm.nih.gov/ entrez/query.fcgi?cmd=Retrieve\&db=PubMed\&list_ uids=23824774\&dopt=Abstract)

20. Baron EJ, Miller JM. Bacterial and Fungal Infections Among Diagnostic Laboratory Workers: Evaluating the Risks. Diagn Microbiol Infect Dis. 2008;60(3):241-6. DOl (http://dx.doi. org/10.1016/j.diagmicrobio.2007.09.016). PubMed (https:// 
www.ncbi.nlm.nih.gov/entrez/query.fcgi?cmd=Retrieve\&db= PubMed\&list_uids=17997259\&dopt=Abstract).

21. Main CL, Carusone SC, David K, Loeb M. Compliance with Personal Precautions Against Exposure to Bloodborne Pathogens Among Laboratory Workers: A Canadian Survey. Infect Control Hosp Epidemiol. 2008;29(1):66-8. DOI (http:// dx.doi.org/10.1086/524325). PubMed (https://www.ncbi.nlm. nih.gov/entrez/query.fcgi?cmd=Retrieve \&db=PubMed\&li st_uids=18171190\&dopt=Abstract).

22. De Carli G, Abiteboul D, Puro V. The Importance of Implementing Safe Sharps Practices in the Laboratory Setting in Europe. Biochem Med (Zagreb). 2014;24(1): 45- 56. DOI (http://dx.doi.org/10.11613/BM.2014.007). PubMed (https://www.ncbi.nlm.nih.gov/entrez/query.fcgi?cmd=Retrieve $\& d b=$ PubMed\&list_uids=24627714\&dopt=Abstract).

23. Shoaei P, Najafi S, Lotfi N, Vakili B, Ataei B, Yaran M, Shafiei R. Seroprevalence of Hepatitis B Virus Infection and Hepatitis B
Surface Antibody Status Among Laboratory Health Care Workers in Isfahan, Iran. Asian J Transfus Sci. 2015;9(2): 138- 40. DOI (http://dx.doi.org/10.4103/0973-6247.162701).

24. Peacock SJ, Schweizer HP, Dance DAB, Smith TL. Gee JE, Wuthiekanun V, DeShazer D, Steinmetz I, Tan P, Currie BJ. Management of Accidental Laboratory Exposure to Burkholderia pseudomallei and B. mallei. Emerg Infect Dis. 2008;14(7):e2. DOI (http://dx.doi.org/10.3201/eid1407.071501). PubMed (https://www.ncbi.nlm.nih.gov/entrez/query.fcgi?cmd=Retrieve\& $\mathrm{db}=$ PubMed\&list_uids=18598617\&dopt=Abstract).

25. Yagupsky P. Brucellae Growing on Thayer-Martin Medium: A Source of Inadvertent Exposure for Laboratory Personnel in Endemic Areas. J Med Microbiol . 2014;63(Pt 1): 148-9. DOI (http://dx.doi.org/10.1099/jmm.0.064121-0). PubMed (https:// www.ncbi.nlm.nih.gov/entrez/query.fcgi? $\mathrm{cmd}=$ Retrieve \&db=Pub Med\&list_uids=24072760\&dopt=Abstract). 


\section{Annexe : Définitions se rapportant à la Loi sur les agents pathogènes humains et les toxines}

\section{Terme}

Agent de la sécurité biologique (ASB) :

Niveau de confinement (NC) :

Zone de confinement :

\section{Exposition :}

Formulaire de suivi de I'exposition :

Formulaire de notification de l'exposition :

Incident :

Laboratoire :

Permis :

Groupe de risque (GR) :

Agents biologiques à cote de sécurité élevée (ABCSE) :

\section{Définition}

Personne désignée pour superviser les pratiques en matière de biosécurité et de biosûreté dans une installation.

Exigences minimales liées au confinement physique et aux pratiques opérationnelles visant la manipulation sécuritaire d'agents pathogènes humains et de toxines dans les laboratoires. Il existe quatre niveaux de confinement, allant du niveau de base au niveau le plus élevé (1 à 4).

Espace physique qui répond aux exigences liées à un niveau de confinement donné. Il peut s'agir d'une salle unique, d'une série de salles situées dans un même endroit ou d'une série de salles adjacentes. La zone de confinement peut comprendre des zones de soutien dédiées, notamment des sas équipés de douches, de vestiaires "propres » et de vestiaires "sales », le cas échéant.

Contact ou proximité étroite avec des agents pathogènes humains ou des toxines pouvant respectivement causer une infection ou une intoxication. Les voies d'exposition sont l'inhalation, l'ingestion, l'inoculation et l'absorption.

Document utilisé pour rapporter et consigner des renseignements liés à une exposition accidentelle préalablement déclarée à l'Agence de la santé publique du Canada, ainsi qu'à l'enquête qui y est associée.

Document utilisé pour déclarer une exposition accidentelle à l'Agence de la santé publique du Canada et pour consigner les renseignements préliminaires associés à cette exposition.

Événement ou situation pouvant causer une blessure, du mal, une infection, une intoxication, une maladie ou un dommage. Les incidents peuvent mettre en cause des matières infectieuses, des animaux infectés ou des toxines. Le déversement, la libération et la perte d'agents pathogènes humains ou de toxines ainsi que l'exposition à ceux-ci, la fuite d'un animal, les cas où un employé se blesse ou développe une maladie, l'accès non autorisé à la zone de confinement, une panne de courant, un incendie, une explosion, une inondation ainsi que toutes les autres situations de crise (p. ex. séisme, ouragan) sont des exemples d'incidents. Les accidents et ceux évités de justesse sont considérés comme des incidents.

Installation même ou aire située à l'intérieur d'une installation dans lesquelles on manipule des matières biologiques à des fins scientifiques ou médicales.

Autorisation délivrée par l'Agence de la santé publique du Canada en vertu de l'article 18 de la Loi sur les agents pathogènes humains et les toxines, permettant de mener une ou plusieurs activités réglementées comportant des agents pathogènes humains ou des toxines. Un permis peut s'appliquer à plusieurs zones de confinement.

Groupe dans lequel les matières biologiques sont classées en fonction de leurs caractéristiques inhérentes, comme la pathogénicité, la virulence, le risque de propagation et l'existence d'un traitement prophylactique ou thérapeutique efficace. Le groupe de risque énonce le risque pour la santé du personnel et du public ainsi que la santé des animaux et des populations animales.

Sous-ensemble d'agents pathogènes humains et de toxines qui présentent un risque accru en matière de biosûreté en raison de la possibilité qu'on les utilise comme arme biologique. Au paragraphe 10 du Règlement sur les agents pathogènes humains et les toxines, les agents biologiques à cote de sécurité élevée sont identifiés comme des agents pathogènes et des toxines " précisés ». Les agents biologiques à cote de sécurité élevée comprennent donc tous les agents pathogènes du groupe de risque 3 et du groupe de risque 4 qui se retrouvent sur la Liste des agents pathogènes humains et animaux et des toxines réglementés à l'exportation, publiée par le Groupe d'Australie et sujette à modifications, à l'exception du virus Duvenhage, du virus rabique et de tous les autres du genre Lyssavirus, du virus de la stomatite vésiculaire, ainsi que du virus de la chorioméningite lymphocytaire. Les agents biologiques à cote de sécurité élevée comprennent aussi toutes les toxines qui se trouvent à la fois à l'annexe 1 de la Loi sur les agents pathogènes humains et les toxines et sur la Liste des agents pathogènes humains et animaux et des toxines réglementés à l'exportation et qui sont présentes en quantités supérieures aux quantités seuils énoncées au paragraphe 10 (2) du Règlement sur les agents pathogènes humains et les toxines.

Pour obtenir d'autres définitions, veuillez consulter la Norme canadienne sur la biosécurité (NCB), Deuxième édition (16). 